\title{
Uncertain pricing decision problem in closed-loop supply chain with risk-averse retailer
}

Hua Ke, Yanzhun Li and Hu Huang*

*Correspondence:

huanghu0213@163.com

School of Economics and

Management, Tongji University,

Siping Road, 200092 Shanghai,

China

\section{黛 Springer}

\begin{abstract}
This paper considers a pricing and remanufacturing problem in closed-loop supply chain which includes one dominant manufacturer and one retailer under uncertain environment. The remanufacturing cost, market demand, and collection cost are characterized as uncertain variables. The firms' optimal strategies are obtained by uncertainty-theory-based and game-theory-based models. Besides, the impacts of the risk sensitivity on the performances of the closed-loop supply chain members are given by the comparison of different degrees of the retailer's risk aversion and numerical studies. It is found that only the manufacturer makes more profits when the retailer is more risk sensitive but this condition in the closed-loop supply chain still leads to better performance of the total system.
\end{abstract}

Keywords: Closed-loop supply chain; Uncertain variable; Remanufacturing; Pricing decision; Risk aversion

\section{Introduction}

With the worldwide promotion of low-carbon economy and sustainable development, closed-loop supply chain management has played an increasingly important role in both the environment and economic activities throughout this decade [1-6]. Reverse collection and remanufacturing are becoming more and more important under this background. Increasing researches about reverse supply chain management have been conducted in this decade [7-11]. For instance, Wei and Zhao [8] addressed the decisions of reverse channel with three different used products collection channels from customers and investigated how the implications of these models affect the decisions of the manufacturer, the retailer, and the third party. Govindan [10] reviewed recently published papers in reverse logistic and closed-loop supply chain in scientific journals and suggested future research opportunities according to these papers. Wei and Zhao [11] considered the pricing and remanufacturing decisions in a duopoly market with two competing supply chains, each of which includes one manufacturer and one retailer. Five game decision models were established to explore the chain members' optimal strategies on price and/or remanufacturing and examined the influences of some key parameters and chain members' maximum profits through numerical studies.

(c) 2015 Ke et al. Open Access This article is distributed under the terms of the Creative Commons Attribution 4.0 International License (http://creativecommons.org/licenses/by/4.0/), which permits unrestricted use, distribution, and reproduction in any medium, provided you give appropriate credit to the original author(s) and the source, provide a link to the Creative Commons license, and indicate if changes were made. 
According to the lack of referable historical data and uncertainties in collection of used products, we adopt uncertainty theory instead of probability theory to solve problems with such variables. For now, uncertainty theory has been successfully applied to many fields, such as option pricing problem [12], stock problem [13], production control problem [14], supply chain pricing problem [15], uncertain random process [16], project scheduling problem [17], and so on. In this paper, we consider the remanufacturing cost, market demand, and collection cost as uncertain variables.

Besides, many researches also take the risk in supply chain into consideration. Agrawal and Seshadri [18] considered a single-period inventory model in which a risk-averse retailer faces random customer demand and decides the selling price with the objective of maximizing expected utility. This research also provided a better understanding of retailers' pricing behavior that could lead to improved price contracts and channel management policies. Barry [19] addressed that the world is at risk and the supply chain is not exempt and raised some essential supply chain questions that have impacts on the field which is from outside of the supply chain. Xiao and Yang [20] developed a priceservice competition model of two supply chains, each including one risk-neutral supplier and one risk-averse retailer to investigate the optimal decisions of players under demand uncertainty and analyzed the effects of the retailers' risk sensitivity on the players' optimal strategies. Ke et al. [21] studied a pricing decision problem in fuzzy supply chain with one manufacturer and two risk-sensitive retailers.

The goal of this paper is to analyze how risk sensitivity affects the performances of the channel members. In consideration of different degrees of the risk aversion of the retailer, two different models are built to derive the optimal management strategies of the supply chain members under different decision scenarios. Moreover, the impacts of the risk sensitivity on the performances of the closed-loop supply chain are also given by numerical studies. It is found that only the manufacturer makes more profits when the retailer is more risk sensitive. Meanwhile, this choice in the closed-loop supply chain leads to better performance of the total system. The results also show how the uncertainty in the supply chain influences the pricing and remanufacturing decisions.

The rest of the paper is organized as follows: Preliminaries are presented in "Preliminaries" section. Some useful notations and necessary assumptions are discussed in "Problem description" section and two uncertain models are detailed in "Models and solution approaches" section. In the "Analysis of the strategy decision" section, numerical experiments are applied to analyze the effects of the uncertainty and power structures on the optimal decisions and maximal profits. Some conclusions are given in the "Conclusions" section.

\section{Problem description}

In this paper, we consider a closed-loop supply chain pricing problem which includes one dominant manufacturer and one retailer and analyze how the retailer's risk sensitivity affects the performances of the channel members. In the forward supply chain, the manufacturer produces new products by using the original component at unit cost $c_{m}$, remanufactures products from used products at unit cost $\tilde{c_{r}}$, which is an uncertain variable, and wholesales the products to the retailer with unit wholesale price $w_{r}$, which is a decision variable. Then, the retailer sells products to the costumer at unit sales price $p_{r}$, 
which is a decision variable. In the reverse supply chain, the used products are recycled from the consumers by different channels.

This paper considers two types of models to distinguish the risk sensitivity of the retailer: (a) expected value (EV) model: both the manufacturer and the retailer are assumed to be risk neutral; (b) chance-constrained (CC) model: the manufacturer is assumed to be risk neutral while the retailer is assumed to be risk sensitive. Within the above two models, we assume that the manufacturer defines the collecting rate $\tau$, $0 \leq \tau \leq 1$. The collector recycles the used products at unit collecting $\operatorname{cost} c(\tau)$. According to Savaskan et al. [3], the collection $\operatorname{cost} c(\tau)$ can be denoted as

$$
c(\tau)=k \tau^{2}+\tilde{p_{c}} \tau q
$$

where $k \tau^{2}$ denotes the fixed expense for recycling used products, $k$ is an elastic parameter, $\tilde{p}_{c} \tau q$ denotes the variable collection cost, $\tau q$ denotes the total number of used products which will be remanufactured, and $\tilde{p_{c}}$ denotes the unit collection cost of used product, an uncertain variable. The notations which will be used in our paper are as follows:

$c_{m}$ : unit manufacturing cost of the product

$w_{r}$ : unit wholesale price of the product, a decision variable

$p_{r}$ : unit sales price of the product, a decision variable

$\tilde{c_{r}}$ : unit remanufacturing cost of the product, an uncertain variable

$\tilde{p}_{c}$ : unit collection cost of used product, an uncertain variable

$\tau$ : the used products collecting rate, $0 \leq \tau \leq 1$

In order to attain the closed-form solutions, some assumptions of this work are made as follows:

Assumption 1. (Linear demand function) Due to the complicated and changeable environment, the demand elastic coefficient cannot be estimated precisely. We define the demand function as a price-dependent linear form

$$
q=\tilde{d}-\tilde{\beta} p_{r}
$$

where $\tilde{d}, \tilde{\beta}$ are uncertain variables, $\tilde{d}$ denotes the primary demand of the new products, while $\tilde{\beta}$ denotes the measure of the responsiveness of the new product's demand to its own price.

Assumption 2. All the uncertain coefficients are assumed nonnegative and mutually independent.

Assumption 3. (Dominant manufacturer) The manufacturer is the Stackelberg leader, and the retailer is the follower under both the two collection models.

Assumption 4. (Risk-averse retailer) The retailer is assumed to be risk averse.

Throughout this paper, we adopt the convention of pronoun "he" to refer to the manufacturer and pronoun "she" to refer to the retailer. The manufacturer in each decision scenario makes his decision to maximize his own expected profit, subjected to the 
retailer's response. We solve the retailer's response function firstly, given that she has observed the manufacturer's decision.

\section{Models and solution approaches}

In this section, Stackelberg game models are employed to derive the equilibrium prices in different scenarios.

\section{EV model}

In this model, the manufacturer dominates the supply chain and first announces the unit wholesale price $w_{r}$ and the used products collecting rate $\tau$. After observing $w_{r}$ and $\tau$, the retailer then decides her most profitable sales price $p_{r}$. Meanwhile, both the manufacturer and retailer are assumed to be risk neutral.

$$
\left\{\begin{array}{l}
\max _{w_{r}, \tau} E\left[\pi_{m}\right]=E\left[\left(w-c_{m}(1-\tau)-\left(\tilde{p}_{c}+\tilde{c}_{r}\right) \tau\right) q-k \tau^{2}\right] \\
\text { where } p_{r}^{*} \text { solves problems: } \\
\qquad \begin{array}{l}
\max _{p_{r}} E\left[\pi_{r}\right]=E\left[\left(p_{r}-w_{r}\right) q\right] \\
p_{r}-w_{r}>0
\end{array}
\end{array}\right.
$$

The retailer's profit function $\pi_{r}\left(p_{r}\right)$ can be denoted as

$$
\pi_{r}\left(p_{r}\right)=\left(p_{r}-w_{r}\right)\left(\tilde{d}-\tilde{\beta} p_{r}\right) .
$$

Then, her expected profit function can be denoted as

$$
E\left[\pi_{r}\left(p_{r}\right)\right]=\left(p_{r}-w_{r}\right) E[\tilde{d}]+E[\tilde{\beta}] w_{r} p_{r}-E[\tilde{\beta}] p_{r}^{2} .
$$

Proposition 1. Under the EV model, the retailer's reaction function, denoted as $p_{d r}$, can be given by considering the manufacturer's decisions $w_{r}$ and $\tau$,

$$
p_{d r}=\frac{E[\tilde{d}]+E[\tilde{\beta}] w_{r}}{2 E[\tilde{\beta}]} .
$$

Under the EV model, the manufacturer's profit function, denoted as $\pi_{m}\left(w_{r}, \tau\right)$, can be given as

$$
\pi_{m}\left(w_{r}, \tau\right)=\left[w-c_{m}(1-\tau)-\left(\tilde{p}_{c}+\tilde{c}_{r}\right) \tau\right] q-k \tau^{2} .
$$

The manufacturer's objective is to maximize his own expected profit $E\left[\pi_{m}\left(w_{r}, \tau\right)\right]$, which can be given as follows

$$
\begin{aligned}
E\left[\pi_{m}\left(w_{r}, \tau\right)\right]= & -k \tau^{2}+\left(w_{r}-c_{m}+c_{m} \tau\right)\left(E[\tilde{d}]-E[\tilde{\beta}] p_{d r}\right) \\
& -\tau \int_{0}^{1}\left(\Phi_{p_{c}}^{-1}(\alpha) \Phi_{d}^{-1}(1-\alpha)+\Phi_{c_{r}}^{-1}(\alpha) \Phi_{d}^{-1}(1-\alpha)\right) d \alpha \\
& +\tau p_{d r}\left(E\left[\tilde{p}_{c} \tilde{\beta}\right]+E\left[\tilde{c_{r}} \tilde{\beta}\right]\right) .
\end{aligned}
$$


Proposition 2. If $k>\frac{\left(E\left[\tilde{p}_{c} \tilde{\beta}\right]+E\left[\tilde{c}_{r} \tilde{\beta}\right]-c_{m} E[\tilde{\beta}]\right)^{2}}{8 E[\tilde{\beta}]}$ holds, the optimal wholesale price, denoted as $w_{d m}^{*}$, and the optimal used products collecting rate, denoted as $\tau_{d m}^{*}$, are given as

$$
w_{d m}^{*}=\frac{A_{2} A_{3}+2 k A_{1}}{2 k E[\tilde{\beta}]-A_{2}^{2}}, \quad \tau_{d m}^{*}=\frac{A_{3} E[\tilde{\beta}]+A_{1} A_{2}}{2 k E[\tilde{\beta}]-A_{2}^{2}}
$$

where

$$
\begin{aligned}
A_{1}= & \frac{E[\tilde{d}]+c_{m} E[\tilde{\beta}]}{2}, \quad A_{2}=\frac{E\left[\tilde{p_{c}} \tilde{\beta}\right]+E\left[\tilde{c_{r}} \tilde{\beta}\right]-c_{m} E[\tilde{\beta}]}{2}, \\
A_{3}= & \frac{E[\tilde{d}]}{2 E[\tilde{\beta}]}\left(E\left[\tilde{\left.p_{c} \tilde{\beta}\right]}+E\left[\tilde{c_{r}} \tilde{\beta}\right]+c_{m} E[\tilde{d}]\right)-\int_{0}^{1}\left(\Phi_{p_{c}}^{-1}(\alpha) \Phi_{d}^{-1}(1-\alpha)\right.\right. \\
& \left.+\Phi_{c_{r}}^{-1}(\alpha) \Phi_{d}^{-1}(1-\alpha)\right) d \alpha .
\end{aligned}
$$

According to Propositions 1 and 2, Proposition 3 can be easily obtained.

Proposition 3. Under the EV model, if $k>\frac{\left(E\left[\tilde{p}_{c} \tilde{\beta}\right]+E\left[\tilde{c_{r}} \tilde{\beta}\right]-c_{m} E[\tilde{\beta}]\right)^{2}}{8 E[\tilde{\beta}]}$ holds, the optimal retail price, denoted as $p_{d r}^{*}$, is given as

$$
p_{d r}^{*}=\frac{E[\tilde{d}]+E[\tilde{\beta}] w_{d m}^{*}}{2 E[\tilde{\beta}]} .
$$

\section{CC model}

The CC model is to maximize the objective function under a certain confidence level and has been widely used to deal with problems with risk-sensitive decision makers.

In this model, the conditions are as same as the former and the retailer is also assumed to be risk sensitive.

$$
\left\{\begin{array}{l}
\max _{w_{r}, \tau} E\left[\pi_{m}\right]=E\left[\left(w_{r}-c_{m}(1-\tau)-\left(\tilde{p}_{c}+\tilde{c}_{r}\right) \tau\right) q-k \tau^{2}\right] \\
\text { where } p_{r}^{*} \text { solves problems: } \\
\left\{\begin{array}{c}
\max _{p_{r}} \bar{\pi}_{r} \\
\mathcal{M}\left\{\left(p_{r}-w_{r}\right)\left(\tilde{d}-\tilde{\beta} p_{r}\right)^{+} \geq \bar{\pi}_{r}\right\} \geq \alpha \\
p_{r}-w_{r}>0
\end{array}\right.
\end{array}\right.
$$

In this paper, we assume that the retailer is risk averse, which means that the confidence level $\alpha>0.5$. To solve the model, we should transform the uncertain model into an equivalent model first.

The equivalent model of the $\mathrm{CC}$ model is as follows:

$$
\left\{\begin{array}{l}
\max _{w_{r}, \tau} E\left[\pi_{m}\right]=E\left[\left(w_{r}-c_{m}(1-\tau)-\left(\tilde{p}_{c}+\tilde{c}_{r}\right) \tau\right) q-k \tau^{2}\right] \\
\text { where } p_{r}^{*} \text { solves problems: } \\
\left\{\begin{array}{l}
\max _{p_{r}}\left(p_{r}-w_{r}\right)\left(\Phi_{d}^{-1}(1-\alpha)-\Phi_{\beta}^{-1}(\alpha) p_{r}\right) \\
p_{r}-w_{r}>0
\end{array}\right.
\end{array}\right.
$$


The retailer's profit function $\pi_{r}\left(p_{r}\right)$ can be denoted as

$$
\pi_{r}\left(p_{r}\right)=\left(p_{r}-w_{r}\right)\left(\Phi_{d}^{-1}(1-\alpha)-\Phi_{\beta}^{-1}(\alpha) p_{r}\right) .
$$

Proposition 4. Under the CC model, the retailer's reaction function, denoted as $p_{d r}$, can be given as follows by considering the manufacturer's decisions $w_{r}$ and $\tau$,

$$
p_{d r}=\frac{\Phi_{d}^{-1}(1-\alpha)+\Phi_{\beta}^{-1}(\alpha) w_{r}}{2 \Phi_{\beta}^{-1}(\alpha)}
$$

Under the CC model, the manufacturer's profit function, denoted as $\pi_{m}\left(w_{r}, \tau\right)$, can be given as

$$
\pi_{m}\left(w_{r}, \tau\right)=\left[w-c_{m}(1-\tau)-\left(\tilde{p}_{c}+\tilde{c}_{r}\right) \tau\right] q-k \tau^{2} .
$$

The manufacturer's objective is to maximize his own expected profit $E\left[\pi_{m}\left(w_{r}, \tau\right)\right]$, which can be given as follows

$$
\begin{aligned}
E\left[\pi_{m}\left(w_{r}, \tau\right)\right]= & -k \tau^{2}+\left(w_{r}-c_{m}+c_{m} \tau\right)\left(E[\tilde{d}]-E[\tilde{\beta}] p_{d r}\right) \\
& -\tau \int_{0}^{1}\left(\Phi_{p_{c}}^{-1}(\alpha) \Phi_{d}^{-1}(1-\alpha)+\Phi_{c_{r}}^{-1}(\alpha) \Phi_{d}^{-1}(1-\alpha)\right) d \alpha \\
& +\tau p_{d r}\left(E\left[\tilde{p_{c}} \tilde{\beta}\right]+E\left[\tilde{c_{r}} \tilde{\beta}\right]\right) .
\end{aligned}
$$

Proposition 5. If $k>\frac{\left(E\left[\tilde{p_{c}} \tilde{\beta}\right]+E\left[\tilde{c_{r}} \tilde{\beta}\right]-c_{m} E[\tilde{\beta}]\right)^{2}}{8 E[\tilde{\beta}]}$ holds, the optimal wholesale price, denoted as $w_{d m}^{*}$, and the optimal used products collecting rate, denoted as $\tau_{d m}^{*}$, are given as

$$
w_{d m}^{*}=\frac{B_{2} B_{3}+2 k B_{1}}{2 k E[\tilde{\beta}]-B_{2}^{2}}, \quad \tau_{d m}^{*}=\frac{B_{3} E[\tilde{\beta}]+B_{1} B_{2}}{2 k E[\tilde{\beta}]-B_{2}^{2}}
$$

where

$$
\begin{aligned}
B_{1}= & E[\tilde{d}]+\frac{c_{m} E[\tilde{\beta}]}{2}-\frac{\Phi_{d}^{-1}(1-\alpha) E[\tilde{\beta}]}{2 \Phi_{\beta}^{-1}(\alpha)}, B_{2}=\frac{E\left[\tilde{p_{c}} \tilde{\beta}\right]+E\left[\tilde{c_{r}} \tilde{\beta}\right]-c_{m} E[\tilde{\beta}]}{2}, \\
B_{3}= & \frac{\Phi_{d}^{-1}(1-\alpha)}{2 \Phi_{\beta}^{-1}(\alpha)}\left(E\left[\tilde{p_{c}} \tilde{\beta}\right]+E\left[\tilde{c_{r}} \tilde{\beta}\right]+c_{m} E[\tilde{d}]\right)-\int_{0}^{1}\left(\Phi_{p_{c}}^{-1}(\alpha) \Phi_{d}^{-1}(1-\alpha)\right. \\
& \left.+\Phi_{c_{r}}^{-1}(\alpha) \Phi_{d}^{-1}(1-\alpha)\right) d \alpha+c_{m} E[\tilde{d}] .
\end{aligned}
$$

According to Propositions 4 and 5, Proposition 6 can be easily obtained.

Proposition 6. Under the CC model, if $k>\frac{\left(E\left[\tilde{p_{c}} \tilde{\beta}\right]+E\left[\tilde{c}_{r} \tilde{\beta}\right]-c_{m} E[\tilde{\beta}]\right)^{2}}{8 E[\tilde{\beta}]}$ holds, the optimal retail price, denoted as $p_{d r}^{*}$, is given as

$$
p_{d r}^{*}=\frac{\Phi_{d}^{-1}(1-\alpha)+\Phi_{\beta}^{-1}(\alpha) w_{d m}^{*}}{2 \Phi_{\beta}^{-1}(\alpha)}
$$




\section{Analysis of the strategy decision}

Due to the complicated forms of the equilibrium prices, numerical examples rather than analytical comparisons are conducted to explore the effects of the risk sensitivity of the retailer on equilibrium prices.

Consider a case in which $c_{m}=40, k=1200, p_{b}=12$, the unit collecting cost $\tilde{p}_{c}$ is about 5 , the unit remanufacturing cost $\tilde{c}_{r}$ is about 20 , the market base $\tilde{d}$ is about 202.5, and the price elasticity $\tilde{\beta}$ is about 8 . These linguistic descriptions of the parameters can be denoted as uncertain numbers as shown in Table 1 .

Referring to Lemma 3, the expected values of the uncertain variables are given in Table 1. For instance,

$$
E[\tilde{d}]=\frac{190+2 * 200+220}{4}=202.5 .
$$

By Lemma 4 and Definition 5, we can have:

$$
\begin{aligned}
E\left[\tilde{p}_{c}^{\alpha} \tilde{d}^{1-\alpha}\right]= & \int_{0}^{1}\left[\Phi_{p_{c}}^{-1}(\alpha) \Phi_{d}^{-1}(1-\alpha)\right] \mathrm{d} \alpha \\
= & \int_{0}^{0.5}(4(1-\alpha)+6 \alpha)(190(1-2(1-\alpha))+2 * 200(1-\alpha)) \mathrm{d} \alpha \\
& +\int_{0.5}^{1}(4(1-\alpha)+6 \alpha)(200(2-2(1-\alpha))+220(1-2(1-\alpha))) \mathrm{d} \alpha \\
= & 1007.5 .
\end{aligned}
$$

Similarly, we can attain the expected values of $E\left[\tilde{c}_{r}^{\alpha} \tilde{d}^{1-\alpha}\right], E\left[\tilde{p}_{c} \tilde{\beta}\right]$, and $E\left[\tilde{c}_{r} \tilde{\beta}\right]$.

The optimal prices and maximal profits of the participants with different degrees of risk aversion are given in Table 2 .

On one hand, we compare the optimal values of the wholesale price, used products collecting rate, and sales price in the CC model and the EV model according to Table 2.

- It is clear that the optimal values of the wholesale price and the collecting rate of used products are becoming higher along with the increase of $\alpha$ in the CC model, which means that the manufacturer can decide higher wholesale price when the retailer is more sensitive to the risk.

- The sales price is becoming lower with the increase of $\alpha$ in the CC model.

- Consumer may prefer that the retailer is risk sensitive because the sales price is highest in the EV model.

On the other hand, the maximal profits of the whole system and individual firms are taken into comparison according to Table 2.

- Both the maximal profits of the whole system and the manufacturer are becoming higher when $\alpha$ increases in the CC model.

- The maximal profit of the retailer is becoming lower when $\alpha$ increases in the CC model.

Table 1 Distributions of uncertain variables

\begin{tabular}{lllll}
\hline Parameter & $\tilde{p}_{c}$ & $\tilde{c}_{r}$ & $\tilde{d}$ & $\tilde{\beta}$ \\
Distribution & $\mathcal{L}(4,6)$ & $\mathcal{L}(19,21)$ & $\mathcal{Z}(190,200,220)$ & $\boldsymbol{Z}(0.7,0.8,0.85)$ \\
Expected value & 5 & 20 & 202.5 & 0.7875 \\
\hline
\end{tabular}


Table 2 Optimal decisions and maximal expected profits

\begin{tabular}{llllllll}
\hline Decision scenario & $\alpha$ & $w_{r}^{*}$ & $\tau^{*}$ & $\pi_{m}$ & $p^{*}$ & $\pi_{r}$ & $\pi_{m}+\pi_{r}$ \\
\hline EV & - & 185.9999 & 0.2807 & 4119.13 & 221.5714 & 996.45 & 5115.58 \\
& 0.6 & 152.8141 & 0.2797 & 5303.43 & 198.6293 & 2111.14 & 7414.57 \\
& 0.7 & 155.4727 & 0.2865 & 5556.37 & 197.2485 & 1970.43 & 7526.80 \\
CC & 0.8 & 158.0673 & 0.2932 & 5808.88 & 195.9011 & 1824.65 & 7633.53 \\
& 0.9 & 160.6000 & 0.2997 & 6060.80 & 194.5857 & 1674.26 & 7735.06 \\
& 1 & 163.0732 & 0.3060 & 6311.95 & 193.3013 & 1519.72 & 7831.67 \\
\hline
\end{tabular}

- The maximal profits of the whole system and individual firms are lowest in the EV model which means that the manufacturer prefers that the retailer is more risk sensitive.

\section{Conclusions}

In this paper, we considered an uncertain pricing decision problem in closed-loop supply chain with risk-averse retailer. The remanufacturing cost, consumer demand, and used products collecting cost were defined as uncertain variables. Uncertainty-theory-based and game-theory-based models were applied to obtain the optimal strategies. The equilibrium behaviors of the participants in the operational level and the optimal decisions on wholesale price, sales price, and used products collecting rate were derived from these models. Numerical experiments were also given to supervise the effects of the retailer's risk sensitivity in strategy level.

Our works mainly focus on the impacts of the retailer's risk sensitivity on the performances of the closed-loop supply chain members with the dominant manufacturer. The different dominant participants and the selections of the recycling channels are the important directions for the future research.

\section{Appendix}

\section{Preliminaries}

In this section, we will introduce some important concepts and theorems of uncertainty theory for modeling the pricing decision problem with human belief degree.

Let $\Gamma$ be a nonempty set and $\mathcal{L}$ a $\sigma$-algebra over $\Gamma$. Each element $\Lambda$ in $\mathcal{L}$ is called an event. The set function $\mathcal{M}$, initiated by Liu [22] and refined by Liu [23], is called an uncertain measure if it satisfies:

Axiom 1. (Normality axiom) $\mathcal{M}\{\Gamma\}=1$.

Axiom 2. (Duality axiom) $\mathcal{M}\{\Lambda\}+\mathcal{M}\left\{\Lambda^{c}\right\}=1$ for any event $\Lambda$.

Axiom 3. (Subadditivity axiom) For every countable sequence of events $\left\{\Lambda_{i}\right\}, i=$ $1,2, \cdots$, we have

$$
\mathcal{M}\left\{\bigcup_{i=1}^{\infty} \Lambda_{i}\right\} \leq \sum_{i=1}^{\infty} \mathcal{M}\left\{\Lambda_{i}\right\} .
$$

Besides, the product uncertain measure on the product $\sigma$-algebra $\mathcal{L}$ was defined by Liu [24] as follows: 
Axiom 4. (Product axiom) Let $\left(\Gamma_{k}, \mathcal{L}_{k}, \mathcal{M}_{k}\right)$ be uncertainty spaces for $k=1,2, \cdots$ The product uncertain measure $\mathcal{M}$ is an uncertain measure satisfying

$$
\mathcal{M}\left\{\prod_{k=1}^{\infty} A_{k}\right\}=\bigwedge_{k=1}^{\infty} \mathcal{M}_{k}\left\{A_{k}\right\}
$$

where $A_{k}$ are arbitrarily chosen events from $\mathcal{L}_{k}$ for $k=1,2, \cdots$, respectively.

Definition 1. [22] An uncertain variable is a measurable function $\xi$ from an uncertainty space $(\Gamma, \mathcal{L}, \mathcal{M})$ to the set of real numbers, i.e., for any Borel set $B$ of real numbers, the set

$$
\{\xi \in B\}=\{\gamma \in \Gamma \mid \xi(\gamma) \in B\}
$$

is an event.

Definition 2. [24] The uncertain variables $\xi_{1}, \xi_{2}, \cdots, \xi_{n}$ are said to be independent if

$$
\mathcal{M}\left\{\bigcap_{i=1}^{n}\left(\xi_{i} \in B_{i}\right)\right\}=\bigwedge_{i=1}^{n} \mathcal{M}\left\{\xi_{i} \in B_{i}\right\}
$$

for any Borel sets $B_{1}, B_{2}, \cdots, B_{n}$.

Definition 3. [22] The uncertainty distribution $\Phi$ of an uncertain variable $\xi$ is defined by

$$
\Phi(x)=\mathcal{M}\{\xi \leq x\}
$$

for any real number $x$.

An uncertainty distribution $\Phi$ is referred to be regular if its inverse function $\Phi^{-1}(\alpha)$ exists and is unique for each $\alpha \in[0,1]$.

Lemma 1. [23] Let $\xi_{1}, \xi_{2}, \cdots, \xi_{n}$ be independent uncertain variables with regular uncertainty distributions $\Phi_{1}, \Phi_{2}, \cdots, \Phi_{n}$, respectively. If the function $f\left(x_{1}, x_{2}, \cdots, x_{n}\right)$ is strictly increasing with respect to $x_{1}, x_{2}, \cdots, x_{m}$ and strictly decreasing with respect to $x_{m+1}, x_{m+2}, \cdots, x_{n}$, then

$$
\xi=f\left(\xi_{1}, \xi_{2}, \cdots, \xi_{n}\right)
$$

is an uncertain variable with inverse uncertainty distribution

$$
\Phi^{-1}(\alpha)=f\left(\Phi_{1}^{-1}(\alpha), \cdots, \Phi_{m}^{-1}(\alpha), \Phi_{m+1}^{-1}(1-\alpha), \cdots, \Phi_{n}^{-1}(1-\alpha)\right) .
$$

Definition 4. [22] Let $\xi$ be an uncertain variable. The expected value of $\xi$ is defined by

$$
E[\xi]=\int_{0}^{+\infty} \mathcal{M}\{\xi \geq r\} d r-\int_{-\infty}^{0} \mathcal{M}\{\xi \leq r\} d r
$$

provided that at least one of the above two integrals is finite.

Lemma 2. [23] Let $\xi$ be an uncertain variable with uncertainty distribution $\Phi$. If the expected value exists, then

$$
E[\xi]=\int_{0}^{+\infty}(1-\Phi(x)) d x-\int_{-\infty}^{0} \Phi(x) d x .
$$


Lemma 3. [23] Let $\xi$ be an uncertain variable with regular uncertainty distribution $\Phi$. If the expected value exists, then

$$
E[\xi]=\int_{0}^{1} \Phi^{-1}(\alpha) d \alpha
$$

Example 1. Let $\xi=\mathcal{L}(a, b)$ be a linear uncertain variable. Then, its uncertainty distribution is

$$
\Phi(x)=\left\{\begin{array}{cr}
0, & x<a \\
(x-a) /(b-a), & a \leq x \leq b \\
1, & x>b
\end{array}\right.
$$

and its inverse uncertainty distribution is $\Phi^{-1}(\alpha)=a+(b-a) \alpha$. The expected value can be attained

$$
E[\xi]=\int_{0}^{1}(a+(b-a) \alpha) \mathrm{d} \alpha=\frac{a+b}{2} .
$$

Example 2. Let $\xi=Z(a, b, c)$ be a zigzag uncertain variable. Then, its uncertainty distribution is

$$
\Phi(x)=\left\{\begin{array}{cr}
0, & x<a \\
(x-a) / 2(b-a), & a \leq x \leq b \\
(x+c-2 b) / 2(c-b), & b<x \leq c \\
1, & x>c
\end{array}\right.
$$

and its inverse uncertainty distribution is

$$
\Phi^{-1}(\alpha)= \begin{cases}(1-2 \alpha) a+2 \alpha b, & \alpha<0.5 \\ (2-2 \alpha) b+(2 \alpha-1) c, & \alpha \geq 0.5\end{cases}
$$

Thus, its expected value is as follows:

$$
E[\xi]=\int_{0}^{0.5}((1-2 \alpha) a+2 \alpha b) \mathrm{d} \alpha+\int_{0.5}^{1}((2-2 \alpha) b+(1-2 \alpha) c) \mathrm{d} \alpha=\frac{a+2 b+c}{4} .
$$

Lemma 4. [25] Let $\xi_{1}, \xi_{2}, \cdots, \xi_{n}$ be independent uncertain variables with regular uncertainty distributions $\Phi_{1}, \Phi_{2}, \cdots, \Phi_{n}$, respectively. A function $f\left(x_{1}, x_{2}, \cdots, x_{n}\right)$ is strictly increasing with respect to $x_{1}, x_{2}, \cdots, x_{m}$ and strictly decreasing with respect to $x_{m+1}, x_{m+2}, \cdots, x_{n}$. Then, the expected value of $\xi=f\left(\xi_{1}, \xi_{2}, \cdots, \xi_{n}\right)$ is

$$
\mathrm{E}[\xi]=\int_{0}^{1} f\left(\Phi_{1}^{-1}(\alpha), \cdots, \Phi_{m}^{-1}(\alpha), \Phi_{m+1}^{-1}(1-\alpha), \cdots, \Phi_{n}^{-1}(1-\alpha)\right) \mathrm{d} \alpha
$$

provided that the expected value $E[\xi]$ exists.

Example 3. Let $\xi$ and $\eta$ be two positive independent uncertain variables with regular uncertainty distributions $\Phi$ and $\Psi$, respectively. Then, we have

$$
E[\xi-\eta]=\int_{0}^{1}\left(\Phi^{-1}(\alpha)-\Psi^{-1}(1-\alpha)\right) \mathrm{d} \alpha .
$$

Definition 5. Let $\tilde{\xi}$ and $\tilde{\eta}$ be uncertain variables. We define 


$$
\begin{aligned}
& E[\tilde{\xi} \tilde{\eta}] \equiv \int_{0}^{1}\left(\tilde{\xi}^{\alpha} \tilde{\eta}^{\alpha}+\tilde{\xi}^{1-\alpha} \tilde{\eta}^{1-\alpha}\right) \mathrm{d} \alpha, \\
& E\left[\tilde{\xi}^{\alpha} \tilde{\eta}^{1-\alpha}\right] \equiv \int_{0}^{1}\left(\tilde{\xi}^{\alpha} \tilde{\eta}^{1-\alpha}+\tilde{\xi}^{1-\alpha} \tilde{\eta}^{\alpha}\right) \mathrm{d} \alpha .
\end{aligned}
$$

\section{Proof of Proposition 1}

Referring to Eq. (5), the first- and second-order partial derivatives of $\pi_{r}\left(p_{r}\right)$ to $p_{r}$ can be shown as

$$
\begin{aligned}
& \frac{\partial E\left[\pi_{r}\left(p_{r}\right)\right]}{\partial p_{r}}=E[\tilde{d}]+E[\tilde{\beta}] w_{r}-2 E[\tilde{\beta}] p_{r}, \\
& \frac{\partial^{2} E\left[\pi_{r}\left(p_{r}\right)\right]}{\partial p_{r}^{2}}=-2 E[\tilde{\beta}]<0 .
\end{aligned}
$$

It follows Eq. (29) that $E\left[\pi_{r}\left(p_{r}\right)\right]$ is concave in $p_{r}$. Setting Eq. (28) to zero, the first-order condition can be given as follows

$$
\frac{\partial E\left[\pi_{r}\left(p_{r}\right)\right]}{\partial p_{r}}=E[\tilde{d}]+E[\tilde{\beta}] w_{r}-2 E[\tilde{\beta}] p_{r}=0 .
$$

By solving Eq. (30), Eq. (6) can be obtained. Proposition 1 is proved.

\section{Proof of Proposition 2}

Referring to Eq. (8), the first-order partial derivatives of $E\left[\pi_{m}\left(w_{r}, \tau\right)\right]$ to $w_{r}$ and $\tau$ can be shown as

$$
\begin{aligned}
\frac{E\left[\pi_{m}\left(w_{r}, \tau\right)\right]}{\partial w_{r}}= & \frac{E[\tilde{d}]+c_{m} E[\tilde{\beta}]-c_{m} \tau E[\tilde{\beta}]+\tau\left(E\left[\tilde{p}_{c} \tilde{\beta}\right]+E\left[\tilde{c}_{r} \tilde{\beta}\right]\right)}{2}-E[\tilde{\beta}] w_{r}, \\
\frac{E\left[\pi_{m}\left(w_{r}, \tau\right)\right]}{\partial \tau}= & \frac{c_{m} E[\tilde{d}]+\left(E\left[\tilde{p_{c}} \tilde{\beta}\right]+E\left[\tilde{c_{r}} \tilde{\beta}\right]-c_{m} E[\tilde{\beta}]\right) w_{r}}{2} \\
& +\frac{\left(E\left[\tilde{p_{c}} \tilde{\beta}\right]+E\left[\tilde{c_{r}} \tilde{\beta}\right]\right) E[\tilde{d}]}{2 E[\tilde{\beta}]}-\int_{0}^{1}\left(\Phi_{p_{c}}^{-1}(\alpha) \Phi_{d}^{-1}(1-\alpha)\right. \\
& \left.+\Phi_{c_{r}}^{-1}(\alpha) \Phi_{d}^{-1}(1-\alpha)\right) d \alpha-2 k \tau .
\end{aligned}
$$

The second-order partial derivatives of $E\left[\pi_{m}\left(w_{r}, \tau\right)\right]$ to $w_{r}$ and $\tau$ can be shown as

$$
\begin{aligned}
& \frac{\partial^{2} E\left[\pi_{m}\left(w_{r}, \tau\right)\right]}{\partial w_{r}^{2}}=-E[\tilde{\beta}]<0, \quad \frac{\partial^{2} E\left[\pi_{m}\left(w_{r}, \tau\right)\right]}{\partial w_{r} \partial \tau}=\frac{E\left[\tilde{p_{c}} \tilde{\beta}\right]+E\left[\tilde{c_{r}} \tilde{\beta}\right]-c_{m} E[\tilde{\beta}]}{2}, \\
& \frac{\partial^{2} E\left[\pi_{m}\left(w_{r}, \tau\right)\right]}{\partial \tau \partial w_{r}}=\frac{E\left[\tilde{p_{c}} \tilde{\beta}\right]+E\left[\tilde{c_{r}} \tilde{\beta}\right]-c_{m} E[\tilde{\beta}]}{2}, \quad \frac{\partial^{2} E\left[\pi_{m}\left(w_{r}, \tau\right)\right]}{\partial \tau^{2}}=-2 k<0 .
\end{aligned}
$$

Then, the Hessian matrix can be attained

$$
\begin{aligned}
& H_{1}=\left|\begin{array}{lll}
\frac{\partial^{2} E\left[\pi_{m}\left(w_{r}, \tau\right)\right]}{\partial w_{r}^{2}} & \frac{\partial^{2} E\left[\pi_{m}\left(w_{r}, \tau\right)\right]}{\partial w_{r} \partial \tau} \\
\frac{\partial^{2} E\left[\pi_{m}\left(w_{r}, \tau\right)\right]}{\partial \tau \partial w_{r}} & \frac{\partial^{2} E\left[\pi_{m}\left(w_{r}, \tau\right)\right]}{\partial \tau^{2}}
\end{array}\right|
\end{aligned}
$$

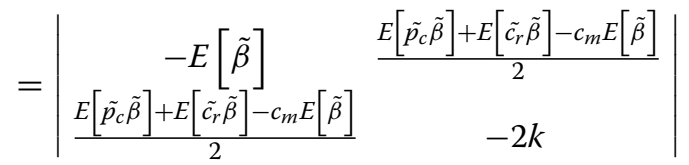


where $H_{1}$ is negative definite when $k>\frac{\left(E\left[\tilde{p}_{c} \tilde{\beta}\right]+E\left[\tilde{c}_{r} \tilde{\beta}\right]-c_{m} E[\tilde{\beta}]\right)^{2}}{8 E[\tilde{\beta}]}$.

Setting Eq. (31) to zero, we can have the first-order condition as follows

$$
\begin{aligned}
\frac{E\left[\pi_{m}\left(w_{r}, \tau\right)\right]}{\partial w_{r}}= & \frac{E[\tilde{d}]+c_{m} E[\tilde{\beta}]-c_{m} \tau E[\tilde{\beta}]+\tau\left(E\left[\tilde{p}_{c} \tilde{\beta}\right]+E\left[\tilde{c_{r}} \tilde{\beta}\right]\right)}{2}-E[\tilde{\beta}] w_{r}=0, \\
\frac{E\left[\pi_{m}\left(w_{r}, \tau\right)\right]}{\partial \tau}= & \frac{c_{m} E[\tilde{d}]+\left(E\left[\tilde{p_{c}} \tilde{\beta}\right]+E\left[\tilde{c_{r}} \tilde{\beta}\right]-c_{m} E[\tilde{\beta}]\right) w_{r}}{2} \\
& +\frac{\left(E\left[\tilde{p_{c}} \tilde{\beta}\right]+E\left[\tilde{c_{r}} \tilde{\beta}\right]\right) E[\tilde{d}]}{2 E[\tilde{\beta}]}-\int_{0}^{1}\left(\Phi_{p_{c}}^{-1}(\alpha) \Phi_{d}^{-1}(1-\alpha)\right. \\
& \left.+\Phi_{c_{r}}^{-1}(\alpha) \Phi_{d}^{-1}(1-\alpha)\right) d \alpha-2 k \tau=0 .
\end{aligned}
$$

Solving Eq. (34), we obtain Eq. (9). Proposition 2 is proved.

\section{Proof of Proposition 4}

Referring to Eq. (14), the first- and second-order partial derivatives of $\pi_{r}\left(p_{r}\right)$ to $p_{r}$ can be shown as

$$
\begin{aligned}
& \frac{\partial \pi_{r}\left(p_{r}\right)}{\partial p_{r}}=\Phi_{d}^{-1}(1-\alpha)+\Phi_{\beta}^{-1}(\alpha) w_{r}-2 \Phi_{\beta}^{-1}(\alpha) p_{r}, \\
& \frac{\partial^{2} \pi_{r}\left(p_{r}\right)}{\partial p_{r}^{2}}=-2 \Phi_{\beta}^{-1}(\alpha)<0 .
\end{aligned}
$$

It follows Eq. (36) that $E\left[\pi_{r}\left(p_{r}\right)\right]$ is concave in $p_{r}$. Setting Eq. (35) to zero, the first-order condition can be given as follows

$$
\frac{\partial \pi_{r}\left(p_{r}\right)}{\partial p_{r}}=\Phi_{d}^{-1}(1-\alpha)+\Phi_{\beta}^{-1}(\alpha) w_{r}-2 \Phi_{\beta}^{-1}(\alpha) p_{r}=0 .
$$

By solving Eq. (37), Eq. (15) can be obtained. Proposition 4 is proved.

\section{Proof of Proposition 5}

Referring to Eq. (17), the first-order partial derivatives of $E\left[\pi_{m}\left(w_{r}, \tau\right)\right]$ to $w_{r}$ and $\tau$ can be shown as

$$
\begin{aligned}
\frac{E\left[\pi_{m}\left(w_{r}, \tau\right)\right]}{\partial w_{r}}= & E[\tilde{d}]-\frac{\Phi_{d}^{-1}(1-\alpha) E[\tilde{\beta}]}{2 \Phi_{\beta}^{-1}(\alpha)}+\frac{\tau}{2}\left(E\left[\tilde{p}_{c} \tilde{\beta}\right]+E\left[\tilde{c_{r}} \tilde{\beta}\right]-c_{m} E[\tilde{\beta}]\right)-E[\tilde{\beta}] w_{r}, \\
\frac{E\left[\pi_{m}\left(w_{r}, \tau\right)\right]}{\partial \tau}= & c_{m} E[\tilde{d}]-\left(\frac{\Phi_{d}^{-1}(1-\alpha)}{2 \Phi_{\beta}^{-1}(\alpha)}+\frac{w_{r}}{2}\right)\left(E\left[\tilde{p_{c}} \tilde{\beta}\right]+E\left[\tilde{c_{r}} \tilde{\beta}\right]-c_{m} E[\tilde{\beta}]\right) \\
& -\int_{0}^{1}\left(\Phi_{p_{c}}^{-1}(\alpha) \Phi_{d}^{-1}(1-\alpha)+\Phi_{c_{r}}^{-1}(\alpha) \Phi_{d}^{-1}(1-\alpha)\right) d \alpha-2 k \tau .
\end{aligned}
$$

The second-order partial derivatives of $E\left[\pi_{m}\left(w_{r}, \tau\right)\right]$ to $w_{r}$ and $\tau$ can be shown as

$$
\begin{aligned}
& \frac{\partial^{2} E\left[\pi_{m}\left(w_{r}, \tau\right)\right]}{\partial w_{r}^{2}}=-E[\tilde{\beta}]<0, \quad \frac{\partial^{2} E\left[\pi_{m}\left(w_{r}, \tau\right)\right]}{\partial w_{r} \partial \tau}=\frac{E\left[\tilde{p_{c}} \tilde{\beta}\right]+E\left[\tilde{c_{r}} \tilde{\beta}\right]-c_{m} E[\tilde{\beta}]}{2}, \\
& \frac{\partial^{2} E\left[\pi_{m}\left(w_{r}, \tau\right)\right]}{\partial \tau \partial w_{r}}=\frac{E\left[\tilde{p_{c}} \tilde{\beta}\right]+E\left[\tilde{c_{r}} \tilde{\beta}\right]-c_{m} E[\tilde{\beta}]}{2}, \quad \frac{\partial^{2} E\left[\pi_{m}\left(w_{r}, \tau\right)\right]}{\partial \tau^{2}}=-2 k<0 .
\end{aligned}
$$


Then, the Hessian matrix can be attained

$$
\begin{aligned}
H_{2} & =\left|\begin{array}{cc}
\frac{\partial^{2} E\left[\pi_{m}\left(w_{r}, \tau\right)\right]}{\partial w_{r}^{2}} & \frac{\partial^{2} E\left[\pi_{m}\left(w_{r}, \tau\right)\right]}{\partial w_{r} \partial \tau} \\
\frac{\partial^{2} E\left[\pi_{m}\left(w_{r}, \tau\right)\right]}{\partial \tau \partial w_{r}} & \frac{\partial^{2} E\left[\pi_{m}\left(w_{r}, \tau\right)\right]}{\partial \tau^{2}}
\end{array}\right| \\
& =\left|\begin{array}{cc}
-E[\tilde{\beta}] & \frac{E\left[\tilde{p_{c}} \tilde{\beta}\right]+E\left[\tilde{c_{r}} \tilde{\beta}\right]-c_{m} E[\tilde{\beta}]}{2} \\
\frac{E\left[\tilde{p_{c}} \tilde{\beta}\right]+E\left[\tilde{c_{r}} \tilde{\beta}\right]-c_{m} E[\tilde{\beta}]}{2} & -2 k
\end{array}\right|
\end{aligned}
$$

where $H_{2}$ is negative definite when $k>\frac{\left(E\left[\tilde{p_{c}} \tilde{\beta}\right]+E\left[\tilde{c_{r}} \tilde{\beta}\right]-c_{m} E[\tilde{\beta}]\right)^{2}}{8 E[\tilde{\beta}]}$.

Setting Eq. (38) to zero, we can have the first-order condition as follows

$$
\begin{aligned}
\frac{E\left[\pi_{m}\left(w_{r}, \tau\right)\right]}{\partial w_{r}}=E[\tilde{d}]- & \frac{\Phi_{d}^{-1}(1-\alpha) E[\tilde{\beta}]}{2 \Phi_{\beta}^{-1}(\alpha)}+\frac{\tau}{2}\left(E\left[\tilde{p_{c}} \tilde{\beta}\right]+E\left[\tilde{c_{r}} \tilde{\beta}\right]-c_{m} E[\tilde{\beta}]\right) \\
& -E[\tilde{\beta}] w_{r}=0, \\
\frac{E\left[\pi_{m}\left(w_{r}, \tau\right)\right]}{\partial \tau}= & c_{m} E[\tilde{d}]-\left(\frac{\Phi_{d}^{-1}(1-\alpha)}{2 \Phi_{\beta}^{-1}(\alpha)}+\frac{w_{r}}{2}\right)\left(E\left[\tilde{p_{c}} \tilde{\beta}\right]+E\left[\tilde{c_{r}} \tilde{\beta}\right]-c_{m} E[\tilde{\beta}]\right) \\
& -\int_{0}^{1}\left(\Phi_{p_{c}}^{-1}(\alpha) \Phi_{d}^{-1}(1-\alpha)+\Phi_{c_{r}}^{-1}(\alpha) \Phi_{d}^{-1}(1-\alpha)\right) d \alpha-2 k \tau=0 .
\end{aligned}
$$

Solving Eq. (41), we obtain Eq. (18). Proposition 5 is proved.

\section{Competing interests}

The authors declare that they have no competing interests.

\section{Acknowledgements}

This work was supported by National Natural Science Foundation of China (Nos. 71371141,71001080).

Received: 12 August 2015 Accepted: 7 October 2015

Published online: 26 October 2015

\section{References}

1. Mentzer, JT, DeWitt, W, Keebler, JS, Min, S, Nix, NW, Smith, CD, Zacharia, ZG: Defining supply chain management J. Bus. Logist. 22(2), 1-25 (2001)

2. Guide, VDR, Van Wassenhove, LN: Closed-loop supply chains: practice and potential. Interfaces. 33(6), 1-2 (2003)

3. Savaskan, RC, Bhattacharya, S, Van Wassenhove, LN: Closed-loop supply chain models with product remanufacturing. Manag. Sci. 50(2), 239-252 (2004)

4. Wassenhove, LNV: The evolution of closed-loop supply chain research. Oper. Res. 57(1), 10-19 (2009)

5. Shi, J, Zhang, G, Sha, J: Optimal production planning for a multi-product closed loop system with uncertain demand and return. Comput. Oper. Res. 38(3), 641-650 (2011)

6. Choi, T-M, Li, Y, Xu, L: Channel leadership, performance and coordination in closed loop supply chains. Int. J. Prod. Econ. 146(1), 371-380 (2013)

7. Prahinski, C, Kocabasoglu, C: Empirical research opportunities in reverse supply chains. Omega. 34(6), 519-532 (2006)

8. Wei, J, Zhao, J: Reverse channel decisions for a fuzzy closed-loop supply chain. Appl. Math. Model. 37(3), 1502-1513 (2013)

9. Zhao, J, Wei, J, Li, Y: Pricing decisions for substitutable products in a two-echelon supply chain with firms' different channel powers. Int. J. Prod. Econ. 153, 243-252 (2014)

10. Govindan, K, Soleimani, H, Kannan, D: Reverse logistics and closed-loop supply chain: a comprehensive review to explore the future. Eur. J. Oper. Res. 240(3), 603-626 (2015)

11. Wei, J, Zhao, J: Pricing and remanufacturing decisions in two competing supply chains. Int. J. Prod. Res. 53(1), 258-278 (2015)

12. Chen, $X$ : American option pricing formula for uncertain financial market. Int. J. Prod. Res. 8(2), 32-37 (2011)

13. Bhattacharyya, R, Chatterjee, A, Kar, S: Uncertainty theory based multiple objective mean-entropy-skewness stock portfolio selection model with transaction costs. J. Uncertainty Anal. Appl. 1(1), 16 (2013)

14. Ke, H: Uncertain random time-cost trade-off problem. J. Uncertainty Anal. Appl. 2(1), 23 (2014)

15. Huang, $\mathrm{H}, \mathrm{Ke}, \mathrm{H}$ : Pricing decision problem for substitutable products based on uncertainty theory. J. Intell. Manuf (2014). doi:10.1007/s10845-014-0991-7

16. Gao, J, Yao, K: Some concepts and theorems of uncertain random process. Int. J. Intell. Syst. 30(1), 52-65 (2015) 
17. Wang, L, Huang, $\mathrm{H}, \mathrm{Ke}, \mathrm{H}$ : Chance-constrained model for RCPSP with uncertain durations. J. Uncertainty Anal. Appl. 3(1), $12(2015)$

18. Agrawal,, , Seshadri, S: Impact of uncertainty and risk aversion on price and order quantity in the newsvendor problem. Manuf. Serv. Oper. Manag. 2(4), 410-423 (2000)

19. Barry, J: Supply chain risk in an uncertain global supply chain environment. Int. J. Phys. Distrib. Logist. Manag. 34(9), 695-697 (2004)

20. Xiao, T, Yang, D: Price and service competition of supply chains with risk-averse retailers under demand uncertainty. Int. J. Prod. Econ. 114(1), 187-200 (2008)

21. Ke, H, Huang, H, Ralescu, DA, Wang, L: Fuzzy bilevel programming with multiple non-cooperative followers: model, algorithm and application. Int. J. Gen. Syst (2015). doi:10.1080/03081079.2015.1086579

22. Liu, B: Uncertainty theory. 2nd edn. Springer, Berlin (2007)

23. Liu, B: Uncertainty theory. 4th edn. Springer, Berlin (2010)

24. Liu, B: Some research problems in uncertainty theory. J Uncertain Syst. 3(1), 3-10 (2009)

25. Liu, Y, Ha, M: Expected value of function of uncertain variables. J Uncertain Syst. 4(3), 181-186 (2010)

\section{Submit your manuscript to a SpringerOpen ${ }^{\circ}$} journal and benefit from:

- Convenient online submission

- Rigorous peer review

- Immediate publication on acceptance

- Open access: articles freely available online

- High visibility within the field

- Retaining the copyright to your article

Submit your next manuscript at $\boldsymbol{\triangleright}$ springeropen.com 